\title{
Three-dimensional Thermal Imaging in Medicine
}

\author{
Adam CHROMY, Ludek ZALUD \\ CEITEC - Central European Institute of Technology, Brno University of Technology, \\ Brno, Czech Republic
}

DOI: $10.15221 / 17.232 \quad$ http://dx.doi.org/10.15221/17.232

\begin{abstract}
The digital medical thermal imaging is valuable qualitative tool for detection of inflamed or necrotic areas, but it lacks the ability of quantification. The new 3D scanning system RoScan composed from robotic manipulator, laser scanner, color camera and thermal imager is presented, extending medical thermal imaging with possibility of quantification, allowing specific $\mathrm{ROI}$ selection and bringing higher thermal resolution in resulting outputs. It helps to solve the current problem of medical quantification, which permeates through the many medical sectors. The advantages of RoScan are demonstrated on sample experiment, which shows the benefits of proposed device.
\end{abstract}

Keywords: 3d body scanning, 3D thermal imaging, thermal quantification, inflammation quantification, medical volumetry, 3D data fusion.

\section{Introduction}

The digital medical thermal imaging (DMTI) is a medical imaging modality bringing the possibility of monitoring the surface temperature of the skin. It was used in first research experimental setups more than 50 years ago, and since then it gradually penetrated to the everyday practice in healthcare domain [1]. The DMTI can be now found in modern clinics on the peripheral vascular, neurological and musculoskeletal conditions assessing and monitoring, in such areas like: cardiology, chronic diseases, dermatology, dentistry, obstetrics, occupational medicine, oncology, physiotherapy, public health, surgery and veterinary medicine [1]-[4].

Due to significant advances in thermal imaging technology, the availability of thermal imagers moved from expensive and bulky systems to compact, affordable and practical solutions [5]. Thermal cameras can be currently found already in smartphones in the price range of up to 700 EUR [6], what makes its reasonable even for small local clinics.

However, despite this advanced spread, this technology is still considered as qualitative tool only, enabling to distinguish between physiological and non-physiological states of body, but without ability to quantify them [7].

The medical quantification itself is a general long-term problem that permeates across the entire healthcare system and that is still not reliably solved. With too inaccurate, insensitive or subjective evaluation methods is struggling, for example, dermatology, traumatology, physiotherapy or many other medical sectors.

Because almost every injury, many diseases or pathological changes are characterized by increased blood flow and stronger cellular metabolic rate in the affected region, such non-physiological states are accompanied with local increase of temperature [8]. From this reason, the DMTI extended with ability of quantification seems to be a suitable tool for medical objective assessment.

In current DMTI, the precise measurements are impossible due to distortions caused by projection of real world to 2D representation (imagine the floor plan of skyscraper - you know the coordinates inside the building but you do not know the floor). Also the region of interest (ROI) is hard to be defined on thermal image due to lack of visibility of added markers.

This paper presents multispectral 3D scanning system RoScan dealing with these drawbacks by data fusion of 3D body scans with color and thermal images, when output 3D models contain besides its spatial representation also the color and temperature of each single point. Thanks to the color layer, the ROI can be precisely selected, the 3D model enables undistorted measurements, and thermal layer exposes non-physiological areas. 


\section{Materials and Methods}

The RoScan system is composed from industrial robotic manipulator, laser 2D profile scanner, industrial color camera and near-infrared camera (thermal imager). All components are connected to the computer that controls the manipulator's movement and acquires images from the sensors (Fig. 1).

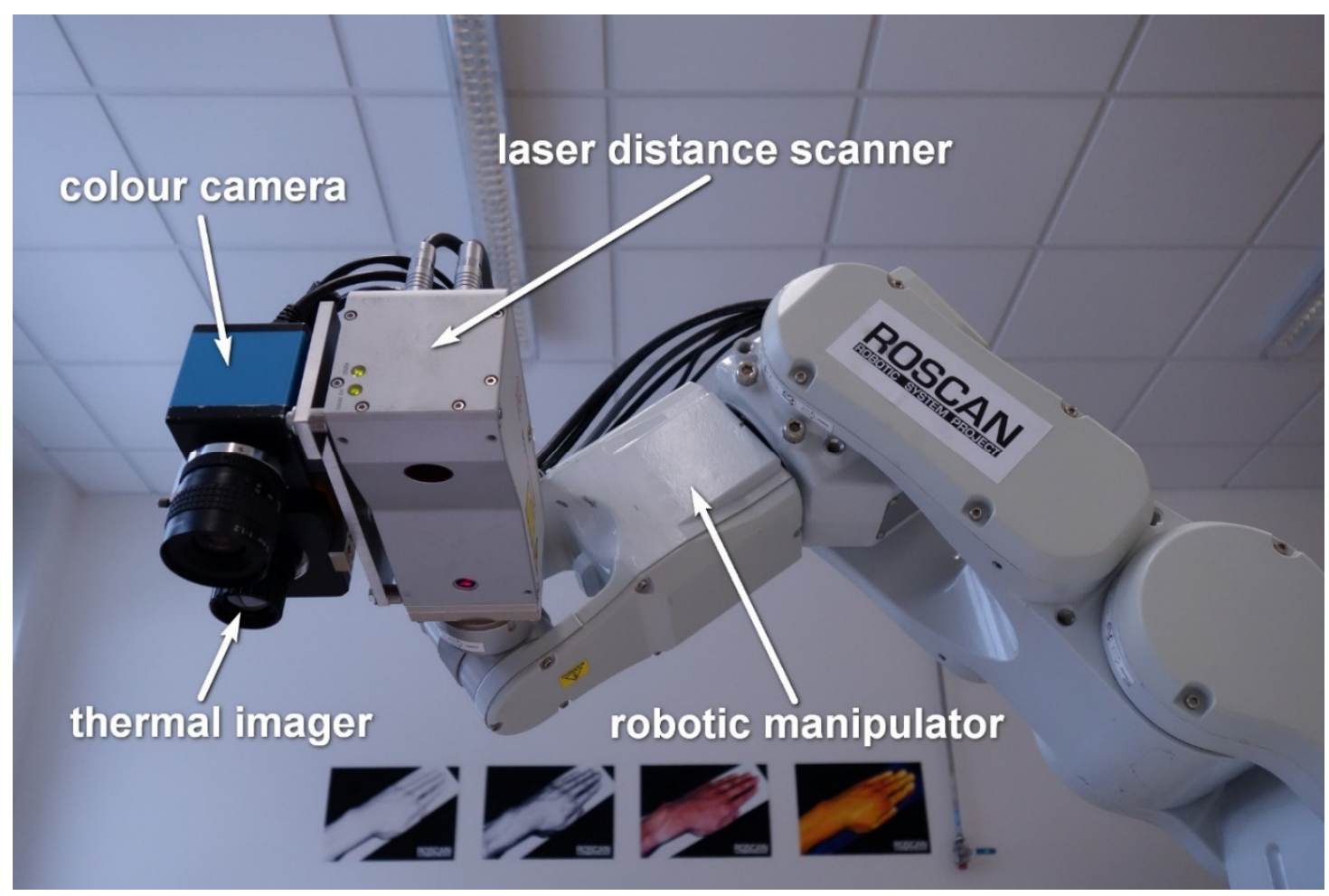

Figure 1 Main components of RoScan system.

The robot used in this device is EPSON C3, which is reaching excellent accuracy of end-point placement $\pm 0.013 \mathrm{~mm}$ [9] and the laser scanner is also very accurate MicroEpsilon ScanCONTROL2750-100 with accuracy $\pm 0.027 \mathrm{~mm}$ [10], what provides overall accuracy ${ }^{1}$ of entire 3D surface scanning system of $\pm 0.12 \mathrm{~mm}[11]$.

Sensoric head is equipped also with LWIR thermal camera Xenics GOBI1954 with resolution $384 \times 288$ pixels, pixel pitch $25 \mu \mathrm{m}$ and spectral response of wavelength range $8 \div 14 \mu \mathrm{m}$ and color camera ImagingSource DFK 51BG02.H with resolution $1600 \times 1200$ pixels.

First, the robotic manipulator moves with the laser scanner around the patient body along predefined trajectory and from several points of view, the data from all three sensors are captured. At each point of scanning trajectory, the laser scanner measures distance to scanned object along measuring line and generates output in form of 2D distance profile. Because we know where the sensor is located at each moment, which direction he looks and how far the laser beam hits the scanned object, we can clearly determine the position of each point in profile. By this process, each captured profile is transformed to the world coordinates and linked with neighboring data into single mesh structure. This entire process, as same as required transforming equations, are deeply described in [12].

Using robotic manipulator empowers both flexibility of movement and high accuracy of captured data, what is usually compromise between these parameters at commercially available scanners [13]. The software, developed for this system, allows definition of custom trajectories composed from geometric primitives using simple scripting language. Such trajectories can be then easily launched for scanning.

After generating 3D model, data from color camera and infrared camera are processed. With each of them we capture several overlapping images, again with recording of where the camera looks and in which direction. Using back beam projection we determine which points in the camera image are related to the corresponding points of the 3D model.

\footnotetext{
1 Term accuracy in this context can be defined as maximal distance between computed (measured) position of point relative to true position of point at $99.7 \%$ of measurements $( \pm 3 \sigma)$
} 
At this point, the ray-tracing algorithm [14] is used, which examine visibility of each point of mesh from camera. If single point is visible from several images, the resulting temperature is given as average of values from these images. The color in such multi-imaged points is given as linear interpolation between colors from these images, weighted by angle relative to 3D surface normal, since the lightness of color is influenced by angle of light reflection. The result is a multi-layer model of the object (Fig. 2).

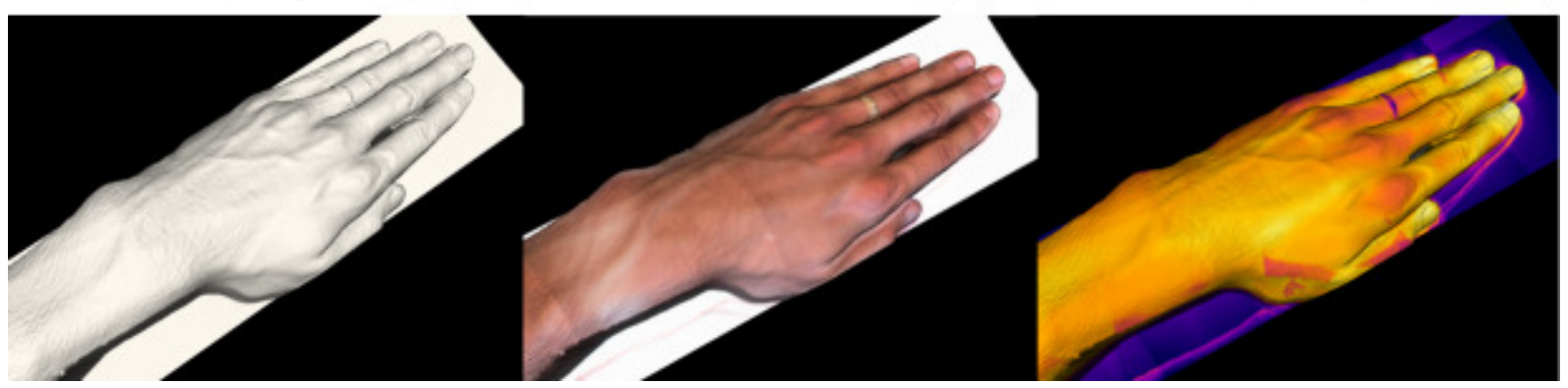

Figure 1 Multi-layered 3D model - the surface (left), color (middle) and temperature (right).

The advantage of this approach is in reaching significantly higher thermal resolution comparing to current DMTI devices, which deals with compromise between resolution and displayed range.

Since ray tracing examination of point visibility is computationally demanding issue, the algorithm uses Octree data structure [15], which divide the area of 3D model into spatial cubes, in which the parts of mesh are classified into. This entire texture mapping process is more deeply described in [16].

Whole scanning process is controlled by our own software (Fig. 3), which is not using any commercial third party components, what makes it arbitrarily adjustable to particular medical application.

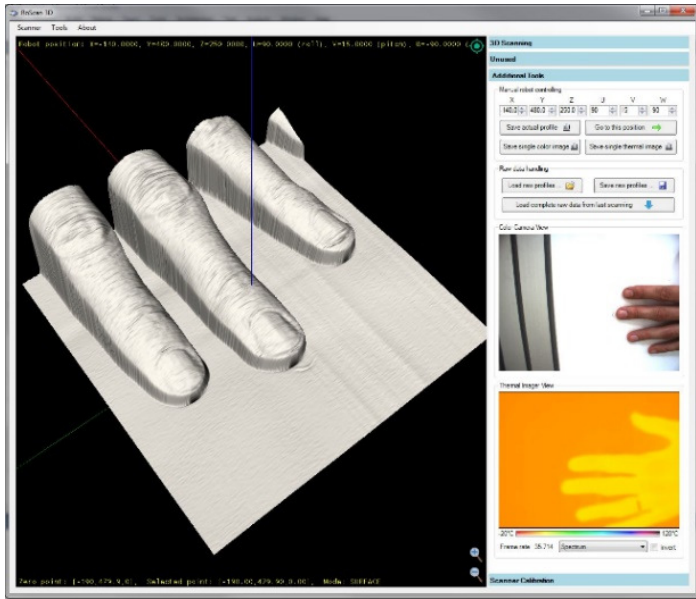

Figure 3 Software for capturing multispectral 3D surface models, screenshot during scanning process.

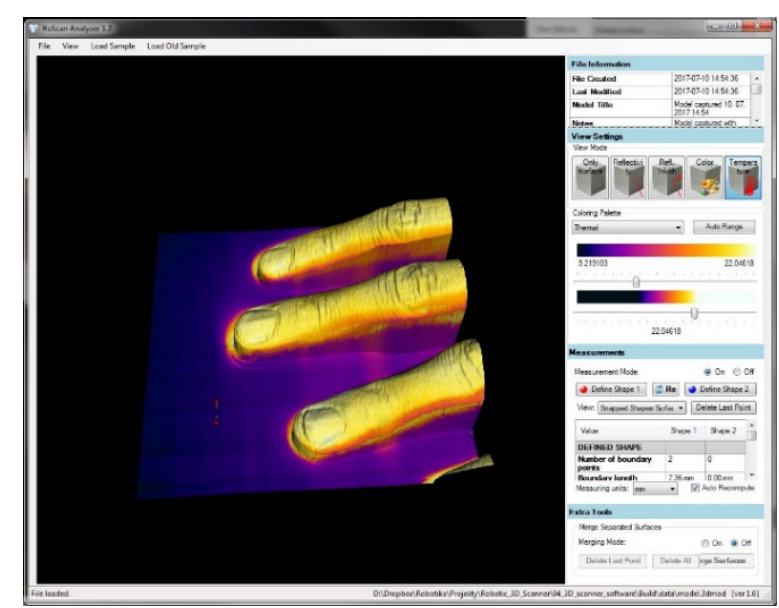

Figure 4 Software for displaying and analyzing multispectral3D surface scans, thermal visualization using false colors.

\subsection{Mutual Calibration of Sensors}

For proper mapping of thermal and color 2D images onto 3D surface model, it is necessary to know the intrinsic parameters of camera very precisely, as same as its 6-DOF position ${ }^{2}$, from which the image has been captured. This position is computed from position of sensoric head and the position of camera relatively to the sensoric head.

In order to estimate these parameters, we use the Bouqet's calibration toolbox [17] implementing Zhang's calibration method [18], which is based on capturing images of calibration pattern from several angles. This method provides intrinsic parameters of camera and extrinsic parameters for each image, which are further compared with location given by robotic manipulator. The pattern is made from PCB with heated copper layer, which is visible on all sensors simultaneously. Calibrating cameras using this method will ensure right mapping of images on the 3D surface. This method is described in [19] with more details.

\footnotetext{
2 6-DOF position means 3 coordinates for camera position and 3 coordinates of rotation, defining the direction of
} camera view. 


\subsection{D Model Analysis}

Captured 3D models can be viewed and analyzed in software tool RoScan Analyzer, which has been developed for th<is purpose (Fig. 4). It provides functionality not only to browse through the 3D models, but also for various measurements of spatial properties of selected regions. It also supports export to standardized formats (e.g. PLY or PTS) for further processing of captured data in other 3D software tools.

It allows showing the scans in 4 different modes:

- Color - Basic view as clinician normally see the patient (Fig. 2 middle). This layer is mostly used for finding the visible landmarks, which are used for orientation (pigmented spots, markers drawn on skin, etc.). Such points can be highlighted using selection markers to be visible also in other layers, or for spatial measurements.

- Temperature - The layer with false colors related to the temperature of skin (Fig. 2 right). The value or particular point can be examined by clicking. Sliders can be used to adjust colortemperature mapping in order to see better the contrast between inflamed and healthy tissue. Because the model is undistorted and properly scaled, the average temperature can be measured according to selected ROI, what would not be possible using common DMTI.

- Roughness - Since we use the laser scanner that is working on triangulation principle [20], besides position of reflected beam ${ }^{3}$, divergence of reflected beam is also measured. This value corresponds to roughness of scanned surface and can be also visualized with false colors. This can be useful when assessing superficial layers of skin in dermatology.

- Surface Only - Sometimes it is important to see some tiny details of the surface (like edges of the scars or boundaries of chronic wound), which are normally hidden in colors (Fig. 2 left). In such cases, only the 3D surface can be displayed, without any coloring.

On each layer, the measurements tool can be activated. Following parameters can be evaluated in selected points or regions of interest (ROI):

- Distances $[\mathrm{mm}]$ - between each two neighboring selection markers on boundary (e.g. length of fingers) or the total length of boundary (e.g. circumference of leg during lymphedema assessment). Distances can be measured either directly or along the shortest path on the surface.

- Angles [deg] - between each three neighboring selection markers on boundary (e.g. vertebrae positions).

- Surface area [mm2] - area of selected ROI (e.g. area of burn) or entire 3D model (e.g. for further computation of burnt percentage).

- Volume [mm2] - volume of selected ROI defined by cutting plane (e.g. volume of swollen finger) or deflected cutting surface (Fig. 4.33 right). ROI can be selected also as internal volume between two selected ROls.

- Color - color of selected point or average color of ROI (e.g. for dermatitis assessments).

- Roughness - provides dimensionless index corresponding to roughness of selected point or average roughness of $\mathrm{ROI}$.

- Temperature $\left[{ }^{\circ} \mathrm{C}\right]$ - temperature of selected point or average temperature of ROI (e.g. for inflammation monitoring).

\section{Results}

Several subjects were examined using RoScan during verification experiments. There were both subjects with physiological state of examined body part and also subjects with inflamed regions. The afflicted regions were clearly visible on captured 3D scans, what proves the usability of RoScan system for diagnostic purposes. In Fig. 5, there is an image of bruised toe after the injury and several hours later. The bruised region is clearly visible in the 3D thermal model.

\footnotetext{
${ }^{3}$ At triangulation laser scanner, the position of reflected beam on detector defines the measured distance.
} 

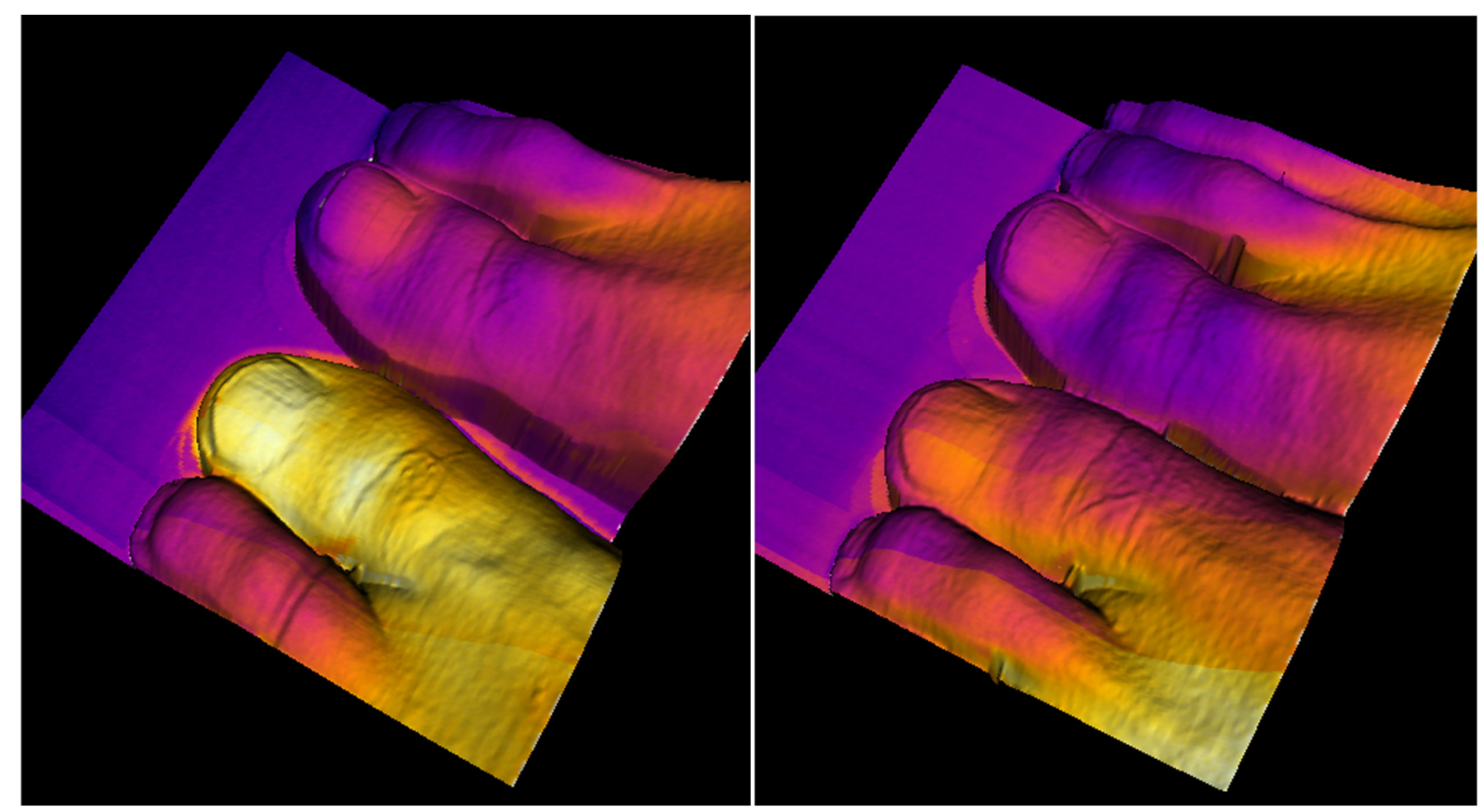

Figure 5 Left: the bumped toe captured 2 hours after injury. Right: Same scene 74 hours after injury. The volume of toe is lower as same as its temperature.

Similar diagnostic data are available also using DMTI, but the exact localization is difficult, since there is lack of clear features in the thermal images and additional markers are not visible. Using RoScan, the inflamed area can be highlighted by selection markers in the thermal layer and then consequently located on color layer, where significant skin landmarks are visible (pigmented sports, etc.) together with additional markers drawn on the skin.

But the exact localization is not the only advantage of RoScan. The major benefit is possibility of objective quantification, the function missing in current DMTI, allowing truthfully record the states during the recovery process.

In Fig. 6, there is a development of volume and average temperature of the bruised thumb on the left foot. The average temperature is lowering almost exponentially during the time, when swelling subsides slowly at the beginning, but faster during the $2^{\text {nd }}$ and $3^{\text {rd }}$ day of recovery.

It is important to note, that at 74 hours after injury (Fig. 5 right), the subject was not already feeling any pain, but thermal difference is still visible and measurable $\left(0.8^{\circ} \mathrm{C}\right)$. This enables early detection of inflamed regions before they are significantly developed, what can bring early treatment, shortening of recovery and treatment costs.

\section{Recovery process of bruised toe}

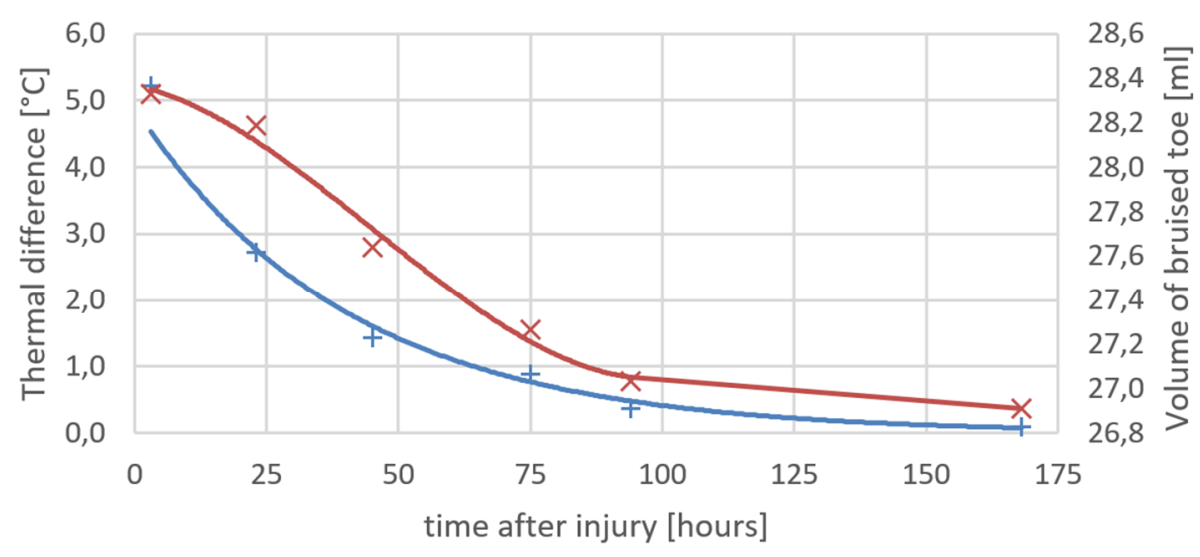

+ Thermal difference between toes $\quad \times$ Volume of bruised toe

Figure 6 Record of temperature development during recovery of bruised toe extended with development of the toe volume. 
Such objective records can be useful for several purposes.

First, the ability of preserve current state of patient objectively is valuable for further comparing during his next visit at the clinician. If the changes are not large enough to be observable, the condition of the patient may be mistakenly considered invariable. It also helps to reliably access the severity of disease, since the method is not as subjective as current ones.

Second, the thermal quantification allows assessment of treatment suitability of medicament efficacy. The selected ROl's can be monitored and changes caused by particular treatment or medicament can be evaluated. For example, the pilot study on topical ointments for atopic dermatitis was performed using RoScan [21].

\section{Conclusions}

The experiments published at [22] already show that DMTI itself seems to be useful diagnostic tool. Its combination with 3D scanners enables also the quantification or objective recording of recovery process, what is currently an issue, which is not sufficiently solved yet and which is actual in many medical sectors. The benefits of creating 3D thermal models were demonstrated on practical experiment with injured subject suffering with bruised toe.

The experiment presented above is not the only application of RoScan in medicine. It has been already used in physiotherapy to localize inflammation center within the ankle, in traumatology for localization and measurement of glass shard encapsulated in the finger or in dermatology to compare efficacy of two different ointments for atopic dermatitis.

RoScan, compared to present methods, brings several advantages. Since the final model is composed from several images, it brings significantly higher resolution of thermal images. Present DMTI devices are limited for compromise between high resolution in tiny displayed area and low resolution in bigger area. RoScan reaches both in the same time.

Combination of 3D model, thermal and color data enables possibility of thermal quantification, what makes RoScan to be a new tool for quantification of inflammation. Since it is capable of selecting precise and specific ROI, even tiny changes, which are not discoverable by present methods, can be now measured. This allows, for example in dermatology, to examine effects of medicament not only in single subject, but also in single lesion, what eliminates influence of subject variance.

The principle of $3 \mathrm{D}$ scanning itself is also advantageous, since precise robotic manipulator enables reaching both high accuracy and advanced flexibility, what is usually trade of in cases of present 3D scanning devices.

The RoScan system is not suitable only for healthcare domain, but also for technical domains. Thermal 3D scanning can be used in electrical engineering for analysis thermal efficiency of electrical components, in civil engineering for monitoring thermal loss of materials or in mechanical engineering for component stress studies.

\section{Acknowledgement}

This work was supported by grant No. 692470, H2020, ECSEL-04-2015-Smart Health, "Advancing Smart Optical Imaging and Sensing for Health (ASTONISH)" and by grant No. FEKT/FIT-J-174745, "Intermodal 3D data registration in healthcare" financed from Internal Science Fund of Brno University of Technology.

\section{References}

[1] R. Vardasca and R. Simoes, "Current Issues in Medical Thermography," in Topics in Medical Image Processing and Computational Vision, Springer, Dordrecht, 2013, pp. 223-237.

[2] D. Hilton-Jones, "Diagnosis and Treatment of Inflammatory Muscle Diseases," J. Neurol. Neurosurg. Psychiatry, vol. 74, no. suppl 2, pp. ii25-ii31, Jan. 2003.

[3] E. F. J. Ring and K. Ammer, "Infrared thermal imaging in medicine," Physiol. Meas., vol. 33, no. 3, p. R33, 2012.

[4] G. Lu and B. Fei, "Medical hyperspectral imaging: a review," J. Biomed. Opt., vol. 19, no. 1, pp. 010901-010901, 2014. 
[5] V. C. Coffey, "Multispectral Imaging Moves into the Mainstream," Opt. Photonics News, vol. 23, no. 4, pp. 18-24, Apr. 2012.

[6] J. T. Hardwicke, O. Osmani, and J. M. Skillman, "Detection of Perforators Using Smartphone Thermal Imaging," Plast. Reconstr. Surg., vol. 137, no. 1, pp. 39-41, Jan. 2016.

[7] X. Ju, J.-C. Nebel, and J. P. Siebert, "3D thermography imaging standardization technique for inflammation diagnosis," in Proceedings Volume 5640, Infrared Components and Their Applications, 2005, p. 266.

[8] T.-C. Chang, Y.-L. Hsiao, and S.-L. Liao, "Application of digital infrared thermal imaging in determining inflammatory state and follow-up effect of methylprednisolone pulse therapy in patients with Graves' ophthalmopathy," Graefes Arch. Clin. Exp. Ophthalmol., vol. 246, no. 1, pp. 45-49, Jan. 2008.

[9] Epson Robots, "Epson C3 Compact 6-Axis RobotManual," 2011. [Online]. Available: http://robots.epson.com/admin/uploads/product_catalog/files/EPSON_C3_Robot_Manual(R7).pdf. [Accessed: 30-Apr-2014].

[10] Micro-Epsilon, "Instruction Manual scanCONTROL," 2008. [Online]. Available: http://www.microepsilon.cz/download/manuals/man--scanCONTROL-2700--en.pdf. [Accessed: 30-Apr-2014].

[11] A. Chromy and L. Zalud, "Robotic 3D scanner as an alternative to standard modalities of medical imaging," SpringerPlus, vol. 3, no. 1, p. 13, 2014.

[12] A. Chromy and L. Zalud, "Novel 3D Modelling System Capturing Objects with Sub-Millimetre Resolution," Adv. Electr. Electron. Eng., vol. 12, no. 5, pp. 476-487, Dec. 2014.

[13] B. Curless, "From Range Scans to 3D Models," SIGGRAPH Comput Graph, vol. 33, no. 4, pp. 3841, Nov. 1999.

[14] K. Suffern, Ray Tracing from the Ground Up. CRC Press, 2016.

[15] T. L. Kunii, Frontiers in Computer Graphics: Proceedings of Computer Graphics Tokyo '84. Springer Science \& Business Media, 2012.

[16] A. Chromy and O. Klima, "3D Scan Model and Thermal Image Data Fusion Algorithms for Three Dimensional Thermal Imaging In Medicine," 2017.

[17] J.-Y. Bouquet, "Camera Calibration Toolbox for Matlab," 2015. .

[18] Z. Zhang, "Flexible camera calibration by viewing a plane from unknown orientations," in Proceedings of the Seventh IEEE International Conference on Computer Vision, 1999, vol. 1, pp. 666-673 vol.1.

[19] A. Chromy, "Mutual Calibration of Sensors for Multispectral 3D Scanning of Surface," 2017.

[20] K. B. Smith and Y. F. Zheng, "Accuracy Analysis of Point Laser Triangulation Probes Using Simulation," J. Manuf. Sci. Eng., vol. 120, no. 4, pp. 736-745, Nov. 1998.

[21] A. Chromy, "Multispectral 3D Surface Scanning System RoScan and its Application in Inflammation Monitoring and Quantification," 2017.

[22] G. Melvin, "Thermography Gallery," Thermal Imaging of the Southwest, 2016. . 